\title{
Gaming after Dark
}

\section{Visual Patterns and Their Significance for Atmosphere and Emotional Experience in Video Games}

\author{
Ivana Müller, Petra Sundström, Martin Murer, and Manfred Tscheligi \\ Christian Doppler Laboratory for Contextual Interfaces, \\ ICT\&S Center, University of Salzburg, \\ Salzburg, Austria \\ firstname. lastnamedsbg.ac.at
}

\begin{abstract}
Design Patterns help a range of designers, architects, and others. However, there is surprisingly little such guidance for game artists. In this paper, we present our look at late 19th century art works and the emergent set of visual features commonly used to create an atmosphere of horror in visual art. Further, we show how we transformed these features into a set of seven patterns to be used in interactive artistry, based on an analysis of six well known survival horror games. Finally, we provide the full description of one of these patterns, the Visual Contrast.
\end{abstract}

Keywords: Design Patterns, Visual Art, Atmosphere, Survival Horror Games.

\section{Introduction}

Game designers and artists constantly encounter new challenges that emerge from the increasing variety of advanced technical upgrades, as well as the demanding expectations of the audience. This has had the effect that the art department often find it hard to keep up. The art often suffers from an uninspired application of these technological, engine-based presets [1]. In this context, a set of visual patterns could also potentially provide the art department with some guidance as to what game engine/technology may be the most suitable for translating a certain style.

One might argue that art is not something that can or should be limited to a set of pre-defined parameters, or that using patterns could diminish creativity and an individual approach to the work performed, but that would be a lack of understanding of what patterns are and in what way they can aid creative process. Patterns are by no means repetitive. They should be considered as hypotheses and/or a collection of ideas, there to act as inspiration and guidance. As every artist would probably agree, it is much easier to be creative if one knows the tools and masters the various methods of expertise.

As a first attempt in this direction, this paper decribes our viewing of an exhibition on uncanny art in Vienna and how the essential aesthetic traits used to evoke a feeling of unease were extracted, adjusted and examined according to how well they mapped 
to six well-known survival horror games. We end this paper by providing an example of how a pattern for visual contrast during enemy encounter in video games can be staged and used.

\section{Background}

Design patterns were first introduced in architectural design by Christopher Alexander [2], but were soon adopted and became a very popular tool in other design-related fields, such as software engineering [e.g. 3], and user experience and experience centered HCI [e.g. 4]. Lately, design patterns have also become an important tool for video game designers, providing many effective problem-solving approaches. The question is, why is there no such help for game artists?

There are, however, tendencies that show that this will happen. For example, Galda from Electronic Arts talked at the FMX'11 conference about their new artistic approach for creating emotionally appealing and cinematic experiences in the game Fight Night Champion. Also, Jonathan Jacques Belletete, the art director of the long awaited game Deus Ex Human Revolution stated, when being interviewed for Gamasutra [5], about their visual concept: “They're motifs. They are patterns. That's the direction we chose". And even if these efforts do nto qualify as visual patterns as of yet, the trend of a structured artistic approach is clear, making this investigation relevant. Introducing the theory of patterns in art could be the basis for another successful brainstorming tool and further, a tool for offering ideas, solutions and methods for a practical translation of artistic concepts into the engine-based 3D space of the game, while preserving significant aspects of art and style.

In terms of already existing pattern-approaches for art, El-Nasr [6] has introduced several patterns for color and lighting techniques for creating the desired visual effect inspired by the staging of style in movies. His patterns were formulated on the basis of a qualitative study of over thirty movies. Such framework can serve as an important additional approach when extending the idea of visual patterns for game designers. Further, Ravaja and colleagues [7] have investigated emotional patterns in video games in terms of sensation seeking. They have also examined emotional responses of joy, relaxation or anger and pointed out that games with different characteristics (different genres) elicit different emotional response patterns.

There are also other works in this direction outside the field of gaming. For example Ståhl and colleagues [8] have explored how shapes, colors and animations can enhance an emotional experience when sending text messages. Also the principles of Gestaltanalysis [9] could be mentioned here as they describe how perception is related to abstractions, symbolism and form.

With the work presented here, our aim is to add to those works and in the longer perspective together form the basis for a set of tools and structures as guidance to game artists. 


\section{An Analysis of Visual Art}

The starting point for this work was an analysis of traditional works of art, which we conducted to derive the key visual features that contribute to an uncanny atmospheric experience in visual art. The horror as used in video games is mainly based on two components; body horror that describes the fear of extreme abnormality and disfigurement of formerly known shapes, and unconscious panic that arises when confronted with the destruction of familiar forms and social aspects. Different from individual personal phobia, these two sources of fear expand beyond the boundaries of society. [10] The analyzed paintings were therefore chosen to cover both of the two themes and even though these paintings were non-interactive, we argue that interactive gaming and interactive art can learn a lot from the more established art traditions.

We looked at several exhibitions. One was Edvard Munch und das Unheimliche, exhibited in 2009/2010 in the Leopold Museum, Vienna. It contained a variety of paintings from Munch, Klinger, Ensor, Kubin and many other painters from the late 19th century, but also offered several essays and concepts from famous personalities, like the psychoanalyst Sigmund Freud and the writer Edgar Allan Poe, both of whom discussed the issue of the uncanny. Different from the usual Christian medieval illustration of horror that was based on portraying death, war or the terrors of hell, artists, writers and psychologist of the 19th century focused on the diffuse fear of obscure emotions and an uncanny atmosphere of the unconscious that, according to them, could be found in everyday life and within our dreams.

\subsection{Visual Features}

To define what visual features to look for, we made use of methods from image analysis [11] as it is applied in traditional art. As our final aim was game play, these features were slightly adjusted, perhaps most apparently in the visual features named Characters and Contrast during Encounter. The visual features we ended up looking for were:

Style. First of all, we analyzed style in general, as it determines basic parameters for all relevant visual factors and decides on the degree of abstraction of the look. Also the visual composition that results from style includes light placement, colors, camera angles, field of view, movement, textures and many more aesthetic features. In games, such art decisions have strong impact on how the game environment is perceived by the players [12]. If the style is well defined and seamlessly pursued throughout the whole work, it helps a lot in experiencing a believable and authentic environment.

Colors, Contrasts and Temperature. The next element we looked at was color and the subsequent scene temperature. Even in 'real' life, we decode at least forty percent of all received information through colors. Colors help us to orientate, to estimate distances and spaces. With nuances of colors, one is able to express the temperature of an image, [13] as well as evoke feelings of ease or unease, pleasure or disgust.

Light. The aspect of lighting was considered next. Light and shadow are inevitable components for creating the atmosphere. They provide clues concerning the spatial 
relationships of objects and reveal, to some extent, the information hidden from the current point of view. [14] Low-key scenarios or strong, dramatic light-effects build tension and expression into the environment. In video games, they also support motion and work as visual guidelines, as the eye reacts rapidly to light-and-dark changes.

Shapes and Geometry. This feature deals with the staging of the environment, based on the principles of geometry. Depending on the overall style, different forms are suitable for providing information about the environment. Cozy and safe rooms are portrayed in completely different shapes than hostile spaces, which employ sharper and edgier shapes.

Characters. Even though paintings show characters or introduce a hero, they are rather impersonal when compared to how they are used in video games, where the character is the main aspect of the story. Defining the looks of the protagonist, as well as various antagonists, is, to a large extent, the task of the art department. It is an essential one, as the player is usually accompanied by the main character throughout the whole game, and the protagonist is the main source of empathy experienced by the player [15]. In survival horror games, the character is usually weak, helpless and left alone in an hostile environment. On the contrary, monsters are creatures that threaten the player's goals and evoke feelings of disgust and fear. Their design usually fits with the style and through their appearance and movement, they directly mirror the attributes of the game atmosphere.

Contrasts during Encounter. This feature is also better represented in games than in paintings, as paintings only can show one single event at a time, while games offer the player a whole palette of visual experiences. This is essential for the changes of atmosphere related to events encountered during gameplay. The visual contrasts during enemy envounter are therefore one of the most subtle yet important aesthetic features in survival horror. This pattern refers to environmental changes that appear throughout the game and is, in other words, the displaying of release and tension through visual manners.

Visual Semantics. Finally, visual semantics deal with special objects, colors, or elements that are easily recognized by the audience as visual leitmotivs and symbolize certain events. Whether they are portrayed in an alienation effect or are an obvious recurring mark, they evoke certain emotional responses. In games, this is a rather easy tool to use, nevertheless a powerful and effective technique for guiding the player's attention and to support the storyline.

To provide an example of a painting we analyzed using the above listed visual features, we present our analysis of Hatred by Pietro Pajetta, painted in 1896. This specific painting was one of the many pieces at the exhibition Edvard Munch und das Unheimliche. The painting presumably deals with the topic of necrophilia and portrays a scene of interfering with the dead. It was chosen because it provides a good example for further game aesthetic analyses, as it not only resembles the semirealistic style of survival horror games, but also introduces characters and distinguished shapes, as often used in game design. 


\subsection{An Analysis of the Painting Hatred}

A representative of the European art scene of the late 19th century, the painting deals with the topic of death in its own subtle yet macabre way. At first sight, the painting shows a man tenderly bending over a woman. Only at second glance can it be noticed that the woman is lying in a coffin and must therefore be dead. The tenderness of the man then seems vaguely strange and leaves the outcome to the viewer's imagination, causing a feeling of unease and anxiety.

Style. The style combines realistic methods with abstracted approaches to underline the topic and to lead the audience's eye to focus on the center of the painting. Even though at first, the painting looks realistically painted, it isn't: The painter carefully focused on the character of the dead woman and added lots of detail to her clothes, especially her white skirt, to make it the most outstanding part of the painting. The second character is almost a mere silhouette, with lack of details. It hovers like an ambiguous shadow over the first character and is nearly as stylized as the nature in the background, which is just implied by its shapes (the trees and the horizon). The level of detail increases with the immediate proximity, while the shapes in the distance appear blurry.

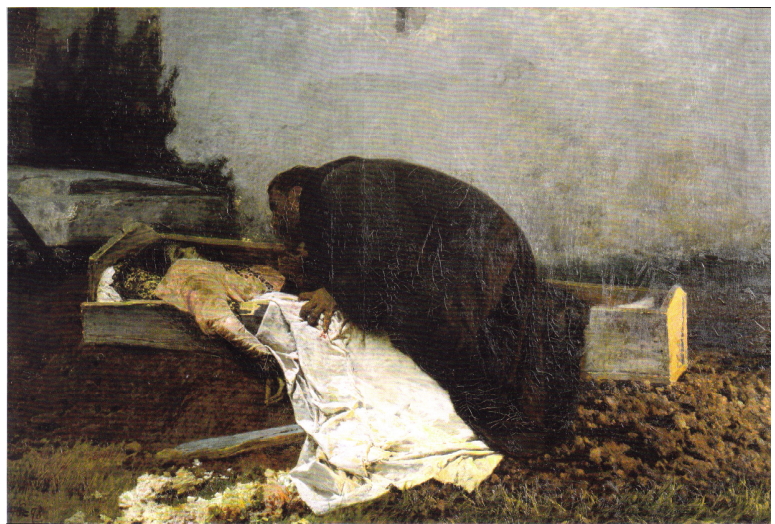

Fig. 1. Hatred by Pietro Pajetta, oil on canvas

Colors, Contrasts and Temperature. The colors used in the painting all share a similar tone of sepia, except for the dark shades of the forest and the figure in the front. The colors used for the background (sky and horizon) are faint and desaturated, while those in the front appear in various shades of rich brown. The only parts that stand out can be found in the shining white skirt of the woman and her slightly dusky pink blouse, which still match the yellowed undertone of the painting. The colors used are not pleasant, as they suggest something rotten, supporting the theme of death.

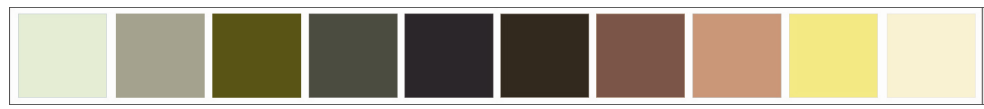

Fig. 2. Color codes as used in Hatred 
The contrasts are visible and distinctive, starting with the most obvious display of the dark, almost black man, bending over the white woman. The two opposite colors touch in an almost exact diagonal that separates the whole painting. The contrasts are visible in the entire image, but especially in the center of the picture, where they are presented by the two characters.

The image also uses contrasts to describe negative spaces: The upper part of the painting, consisting of the lighter background (horizon and sky) serves as contrast to the dark silhouette of the man, while the lower part is almost the exact counterpart of this display, showing it as a negative (in reverted colors). See Figure 3.

The colors also play an important role for displaying the distance within the painting (apart from the contrasts of dark proximity and light background). The closer the scene is to the audience, the warmer the colors become and the more the shades of sepia take over.
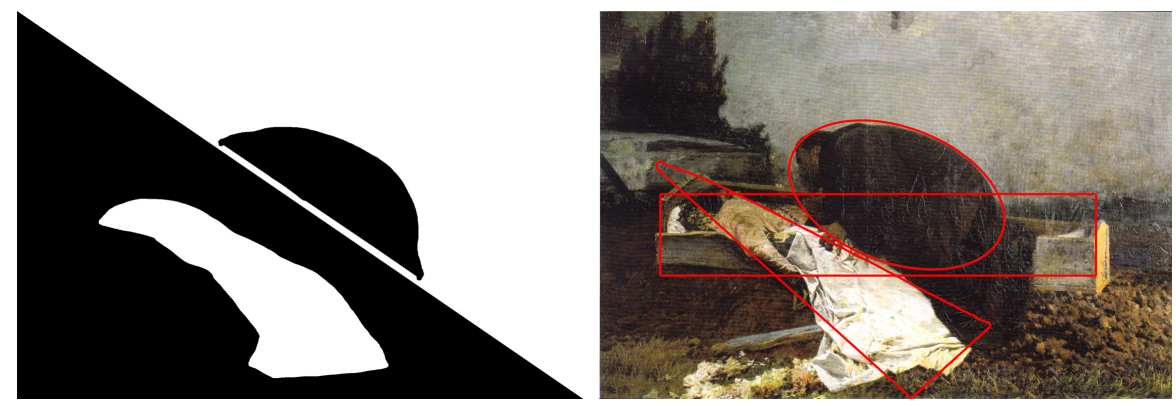

Fig. 3. Contrasts and Geometry as used in Hatred

Light. The light in the painting is flat and non-realistic. While the sky suggests dusk or late dawn, the lighting is different than it should be at twilight. The warm light coming from the right could be explained by an additional light source not visible in the image, but the way the dead woman is illuminated is reminiscent rather of the surrealist approach to lighting. While every other element within the painting is dark, the figure of the woman is overexposed.

Shapes and Geometry. The painting works strongly with essential geometric shapes, see Figure 3. Each character or object represents one shape and the nature divides the image into two sections. Additionally, all basic shapes are in the very center of the piece. The shapes of the two characters are interesting; the man is represented by a circle (or rather an oval/egg-shaped form), while the woman with her dress is positioned as a triangle. While round shapes usually suggest safety and cosines, the man is dark and appears unpleasant, even dangerous. On the other hand, the unusual shape of the dead woman suggest her vulnerability, as she is lying there, unbent and stiff, directly exposed to the dark mass of the man, hovering over her body.

Characters. The painting introduces two characters, contradicting in many aspects: first, there is the body of a dead woman, shown in faint, pastel colors and strongly illuminated. Compared to her counterpart, she suggests something fragile and pure, 
while the second character appears dark and dangerous, threatening her peace. She is remarkably detailed, whereas the second character, the mysterious man, is painted in dark, undistinguished colors, reminiscent of a huge shadow. Even though he may look gentle at first sight, the colors and the context presents him as doubtlessly malevolent.

Visual Semantics. Not only the colors, light and contrast, but also the choice of the characters themselves suggests a duality portrayed in the painting. Starting with obvious differences, such as the bold use of black and white, the symbolism is carried on with the contrariety of man and woman and taken a step further, the dichotomy of the dead and the living.

About ten paintings were analyzed this way. This gave us a collection of visual features used for illustrating the themes of horror and fear. We then set ourselves the task of analyzing how well these features already were used in interactive gaming, and also to look for other tools and features that we had not seen used in the paintings.

\section{An Analysis of Six Survival Horror Games}

Six famous survival horror games were chosen for our analysis of how well these visual features were already used (and if they were used at all) in successful game titles; three western and three Japanese (or eastern, but incidentally, all three were Japanese). These games were chosen after interviews with 14 people who consider themselves fans of the survival horror genre. We asked them about their preferred games, as well as games they experienced as extraordinarily frightening. Apart from these interviews, we also looked at several online communities ${ }^{1}$ devoted to the subject and looked at their top lists for the most popular games in the genre. The final selection was made according to how uncanny these games were considered to be, and to their use of body horror and unconscious panic. The final set of games was:

Dead Space 1. Developed by the U.S. studio Visceral Games and EA Redwood Shores and published by EA in 2008, this 3rd person shooter game follows the storyline of Isaac Clarke, an engineer who has to survive on board of the USG Ishimura, an interstellar mining ship infested with mutant human-like creatures called Necromorphs.

Silent Hill 2. Konami's survival horror classic from 2001 that relies mainly on dynamics of aberrant psychology as its most essential element, was the second game analyzed. The game evolves around the protagonist James who receives a letter from his late wife Mary, inviting him to meet her at their special place in a small town called Silent Hill. As soon as James arrives there to solve the mystery, his nightmarish journey through distorted realities and obscure locations begins, constantly walking the thin line between sanity and madness.

Alan Wake. A story-driven survival horror game, developed by the Finnish studio Remedy Entertainment and published by Microsoft Game Studios in 2010, was the

\footnotetext{
${ }^{1}$ Gamasutra.com (with various contributions), Dreamdawn.com, Compactiongames.about.com, Joystickdivision.com, Vsrecommendedgames.wikia.com, Thegamershub.net, Gameranx.com
} 
third game we looked at. This game skillfully adapts the strengths of psychological thrillers with a cinematic look and feel. The plot follows Alan Wake, a novel writer on his journey to uncover the mysteries of the small town Bright Falls in Alaska and investigate the disappearance of his beloved wife. The main idea of the game is to fight darkness with the use of light.

Amnesia - The Dark Descent. The third western game looked at, developed and published by Swedish Frictional Games in 2010, features the unarmed protagonist named Daniel, suffering from amnesia. The player guides the character through a dark and foreboding castle, avoiding terrible creatures and dangerous obstructions, while trying to regain his memory.

Project Zero (Fatal Frame). The Japanese survival horror game named Project Zero, but known as Fatal Frame in the USA, was released in 2001 by Temco. It has a unique plot that deals with ghosts, exorcism and dark, evil Shinto rituals. The game follows the young girl Miku Hinasaki on the search for her missing brother. Equipped only with an old camera, the player must survive among lost, hostile souls and make his/her way through a haunted mansion.

Haunting Ground (Demento). This highly disturbing survival horror game developed and published by Capcom in 2005 was the last of the Japanese games we looked at. The plot revolves around Fiona Belli, who awakens in a sinister castle after surviving a car accident. Even though her memories seem too vague to figure out what happened, she soon realizes that she must quickly escape from the castle. Accompanied by a German Shepherd, Fiona tries to evade and hide from the odd personnel of the castle that is stalking and hunting her down, trying to kill her.

Each of the six games was analyzed using the list of visual features defined above. For that purpose, we sat next to persons playing these games and took notes while talking to the players about their experiences in various game-play situations. Our focus was on the aesthetic display of the game. Due to space limitations, this paper only presents the analysis of one of the games, Alan Wake.

\subsection{The Analysis of the Game Alan Wake}

Style. This game pursues an almost photorealistic look that supports the cinematic approach. It fits well with the concept of displaying the journey of Alan as TV- like episodes. Due to its overwhelming visual style, the game is very touching and immersive and draws the player gradually into the world of Alan Wake with an intensity that goes under the skin.

Colors, Contrasts and Temperature. The use of colors is intuitive; see Figure 4 for the color code. As the change of day and night plays a crucial role in the game, the scenes change, not only in terms of color, but also in contrast, hue and saturation. The night is basically deep blue, with highly saturated tones of indigo. It has passages of pure black and the contrast is set high. Overall, the night conveys an intense color experience. However, once inside a building, there are almost no traces left of the strong saturation. The colors, even though basically staying the same bluish teints of 
violet or green, seem to be washed out, grey rather than colorful. There are also shades of sepia mixed with the colors. Still, the shadows remain deep black, providing a hard contrast between fair and dark areas. The daylight, on the other hand, appears colorless in comparison to the night. The exterior is very bright with low contrast, mainly filled with shades of sepia and grey, both in very low saturation. The interior is similar, just a little bit darker and denser, reminiscent of the color codes of other survival horror games. The illustrative change starts with the dusk, when darkness approaches. First of all, the contrast settings improve even though the hues do not change. Dark areas filled with grey shadows become deep dark and seem to expand. After that, the hues take on shades of violet and brown with a higher saturation that evokes an eerie feeling, and finally the intense blue hue merges with the other colors.

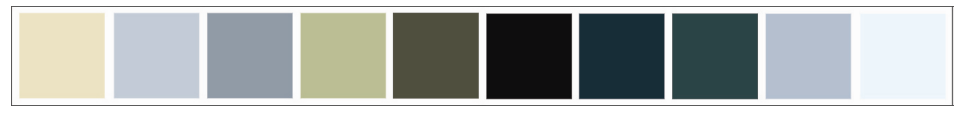

Fig. 4. Color codes as used in Alan Wake

Light. The lighting also depends on how day and night changes in this game. The light during the night almost always outshines its source and causes strong rimlights to accentuate silhouettes. On the contrary, shadows appear deeply dark, in both static and moving conditions. Glowing spots and haze in the background signify darkness. Again, daylight is extremely radiant and almost purely white. Even though it should improve the feeling of security during a sunny day, the fact that the light is colorless and white makes it appear sterile and cold, almost without any shadows at all. The third aspect of light in this game is related to the torchlight that is an indispensable tool for Alan. It provides the protagonist with an enhanced view in darkness and is essential during the fights against dark creatures. The emblematic cone of light that shines down on Alan from time to time is displayed by dense, strong light, piercing through darkness, shielding and protecting him.

Shapes and Geometry (Environment). As the setting is the small town of Bright Falls, the game world is open and provides the player with interiors as well as open landscape with forests, lakes and meadows. The nature is shown in a beautiful, almost poetic way, while turning vile and wild when roamed by the Dark Presence. The buildings are rustic and country-style, reminding one that the town used to be an Indian colony.

Characters (and Monsters). The protagonist Alan Wake is a very natural looking character with a likable face. It is easy to empathize with him and even become attracted to his looks. The main antagonist in the game is known as the Dark Presence and is a dark and evil force responsible for the nightmares haunting Bright Falls. Visually, the Dark Presence is manifested as heavy fog, bleach smoke, dense shadow, swarms of birds or even swarms of people. The player encounters the Dark Presence mostly as dangerous dark, humanoid, faceless creatures, immortal unless pierced by the torchlight. 
Contrast during Encounter. The contrast during an encounter with enemies is a visually impressive experience. The whole screen changes color and fades to apparently unrelated shades. The colors become expressive in hue and saturation, shifting between blue-green, blue-violet and red or pink. Any light sources are strongly outshined, adding glowing and sparkling accents to the scene. The shadows appear more vivid, dancing around in wild motion. At the same time, the player is exposed to slow motion, motion blurs and bloom post effects, while shiny sparks emit more confusing lights. The camera is shaking and when hit, the whole screen turns red. The signs of enemy approaching include exploding light sources that can be noted immediately before the actual encounter.

Visual Semantics. The main theme of Alan Wake is light and darkness, and not only in terms of daytime vs. nighttime, but also in terms of mental sanity. This is visualized through visual responses within the game: the day is desaturated, almost colorless and faint, and the night nearly bursts in intense colors. It is during the night that Alan must pursue his quest and during daytime that he has to face his challenges with advancing logics that evoke doubts about his mental state and sanity, dealing with his nightly encounters with the Dark Presence.

In addition to the analysis of the six games, we watched several walkthrough-videos of each of these games, put online by various players. As many of the walkthrough videos were commented, we could easily identify the moods and anxieties various players were experiencing during the game, even though we never were in direct contact with any of the players. This resulted in descriptions of each of the games, same as the one presented above for Alan Wake.

\section{$5 \quad$ Visual Patterns}

In the final step, we extracted the information about each of the visual features from the six descriptions we had obtained by game analysis. These extractions were then summarized in the basis of their similarities and adjusted into a pattern description along the lines of the pattern template as used in game design [16]:

Name. A single word or a short phrase, defining the main concept of the pattern.

Core Definition. A brief sentence describing the core content of the pattern. The idea behind core definitions is to provide an initial overview for browsing through an extensive pattern collection.

General Description. A short, general description of properties that provide the fundamentals of the pattern, as well as the motivation for its name. General properties are stated by using game examples and concepts that occur in the component framework. The general description is concluded by some explicit examples of various aspects and games that contain the pattern.

Use of the Pattern. A variety of choices which can be helpful when applying the pattern are listed.

Consequences. This part provides information about issues that may appear when a pattern is found in a game. For our purposes, this section is directed toward analyzing 
visual aspects to help solve artistic problems in an already existing visual design, or to suggest other patterns to instantiate in the art of the game.

Relations. This section lists the relations that exist between the analyzed pattern and other patterns. Basically all patterns occuring in any game design concept are in relation to each other, due to the interactivity of a game. Any related pattern is sorted into five categories with different kinds of relations: Instantiates (meaning the presence of the pattern requires the presence of a second pattern), Modulates (the pattern changes certain aspects and features of a second pattern), Instantiated by (the pattern can be instantiated by the presence of a related pattern), Modulated by (if additional patterns are applied, this will happen to this specific pattern) and Potentially conflicted with (using this pattern makes the use of another pattern impossible).

Examples. Here, the direct inspirations for creating the pattern are listed, as well as some descriptions of the main aspects of the pattern.

Due to space limitations, we can only present one pattern we created in this paper. Also, this paper is mainly concerned with the proces of creating patterns and the arguments for using them, and not so much with the patterns themselves (that would be a different kind of publication). The pattern Visual Contrast during Encounter was chosen to represent the created patterns, as it not only combines various aesthetic aspects, such as light and color, but also refers to important components of interactivity essential for the game experience. The other six patterns emergent from our work were: Style, Color, Light, Geometry, Characters and Visual Semantics.

\subsection{The Pattern for Visual Contrast during Encounter}

Core Definition. This pattern describes how to apply visual changes to the overall style during certain events within the game, to guide the player's attention and increase immersion.

General Description. Visual Contrast during Encounter with enemies is strongly connected to tension during gameplay, as (especially in the survival horror genre) the player is left with doubts about the outcome of the battle and the game at large. Emotional cues during such moments are expressively visualized in various ways in order to emphasize the fact that the player is not fully in control of the events and more importantly, of his/her character.

Example from Haunting Ground. When encountering an enemy stalker during gameplay, Fiona, the heroine of Haunting Ground, may panic. Her vision becomes limited to a few essential things, her focus shifts and colors change in contrast and saturation. Her movements are restricted by adding a freeze-frame effect, combined with strong motion blur and camera shakes.

Using the Pattern. The presence of enemies, deadly traps as well as player character killing and player character elimination are the basis for the existence of factors attempting to inflict damage on the player and thus create a feeling of tension. In the survival horror genre, the player expects these factors all the time, which puts him/ her in a constant state of tension. This feeling can be even improved by the actual encounter with such a factor - usually in the form of an enemy. Encounters are a strong visually impressive experience for the player and have two main purposes: on 
the one hand, it is important to visually emphasize the immediate danger coming from the encounter and urge the player to change the situation, whether it is through fighting or running; on the other hand, they strengthen the connection between the protagonist and player, as the protagonist's fears are directly communicated, showing his/her limited vision and abilities caused by the panic s/he experiences.

Making use of various elements to support this situation, the image is filled with disturbing visuals, such as swaying and shaking of the camera, change of saturation and hue, stronger contrast and often also movement of lighting, combined with additional light sources that increase spaces of lit areas, and darkest shadows. The survival horror genre introduces some key features, such as the third-person perspective (in most cases) that allows for the showing of the scenery framed in high-or low angle long shots or distorted lenses in order to increase the feeling of vulnerability and isolation and especially to enhance the shock experienced during an encounter. These features add dramatic tension to the scene, allowing the player to see things that would usually remain lost, such as the approach of monsters and the protagonist running for his/her life at the same time. The tension of an encounter reaches its visual peak when the monster is able to grab the protagonist - this is the moment when the character usually panics the most. Often, this moment is displayed in a short negative shot - an image with reversed tones - followed by the most advanced dissociation of the usual look and feel. The camera might move around way too fast or in very slow motion, while the character and the monster's movements can appear excessively jerky, with the overall representation of the horrific game world possibly becoming very confusing.

Consequences. The visual representation of an encounter serves mainly the purpose of shifting the focus of the player, directly influencing the perception of the horrific atmosphere when experiencing loss of control. Considerations of a probable threat lead to a state of constant emotional anxiety which is a good base for experiencing shock, as this exact scenario is more or less expected by players of survival horror games. According to Power and Dalgleish [17], the fear reactions are mainly triggered by expecting the threat, rather than observing it. The immediate encounter is the climax of such expectations, resulting in the peak of a visually disturbing alienation. The entire graphic representation focuses on communicating the experience of shock and loss of control to the player; it integrates elements of cinematography, light and color as well as multiple post effects within the scene in order to change the image to its utmost expressive and vertiginous extent. The pattern for Visual Contrast during Encounter points out which visual elements are essential for triggering both emotions: the anxiety that slowly grows when the player starts to suspect hostile changes within the environment, as well as the initial shock that is experienced when the confrontation with the long-expected fearsome enemy actually happens. Apart from this conceptual inspiration, the pattern also offers suggestions about the use of different tools and techniques to achieve the desired emotional response within the game, such as post effects, camera shakes, animation of lights, desaturation or shifting of colors or freeze-frame animation. In terms of lighting for improving tension, several game titles already embraced the utility of cinematic lighting effects for projecting peaks of excitement and fear in gameplay.

"Examples include flickering lights interjected at specific moments in the game, darkly lit environments or the use of saturated red." [6] 

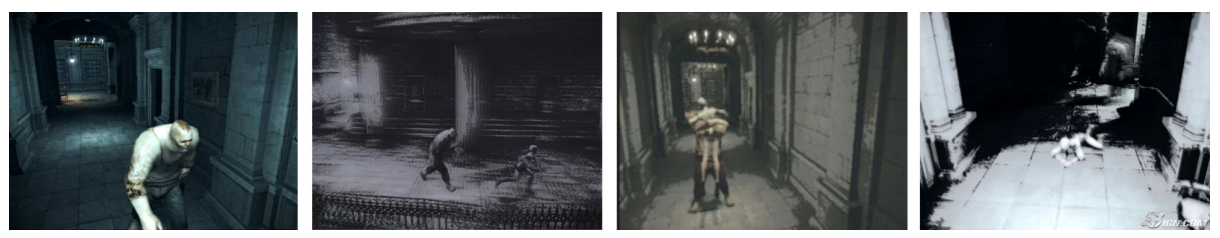

Fig. 5. Visual Contrast during Enemy-Encounter in Haunting Ground

\section{Relations $^{2}$}

- Instantiates: Emotional Immersion, Tension, Disruption of Focused Attention, Fear, Emotional Response, Visual Acceptance, Field of Vision

- Modulates: Focus of Attention, Perception of Environment, Imperfect Information, Player Behavior, Character Behavior

- Instantiated by: Monsters, Penalties, Combat, Damage

- Modulated by: Time Limits, Character Killing, Player Behavior

- Potentially conflicting with: Visual Style, Gameplay

\section{Conclusion}

This paper outlines our attempt to take the first steps towards the creation of a set of visual patterns for game artists. We took visual features obtained by image analysis, and used them as a basis for an analysis of the manner in which visual art conveys an atmosphere of horror. Then, we explored how it is used within game design. We used these findings to generate a set of seven patterns specifically for the survival horror game genre, with the pattern for Visual Contrast during Encounter included in this paper. More research and experiments are needed in order to formulate a working collection of visual patterns to enhance the game art workflow in terms of concept and production. Visual patterns for genres other than survival horror are, of course, also necessary to be created and worked on. It is important to note that different genres will stimulate different visual clues, and thus different emotional responses within the game [e.g 18, 19] and result in a variety of different visual patterns; however, the overall aim remains the same - visual patterns should provide game artists with:

1. Inspiration and overview of artistic approaches to use within the specified genre.

2. A wide range of possibilities and tools to translate a visual direction into technologies and game-engines that are likely to be used without losing the genuine artistic approach to the engine's technology.

\footnotetext{
2 The Relations-section was added to the pattern for the sake of completeness. It will become more relevant when used with the set of future visual patterns that influence and relate to eachother, just as described above.
} 
Acknowledgements. We greatly acknowledge the financial support by the Federal Ministry of Economy, Family and Youth and the National Foundation for Research, Technology and Development (Christian Doppler Laboratory for "Contextual Interfaces") and the project "AIR - Advanced Interface Research" funded by the Austrian Research Promotion Agency (FFG), the ZIT Center for Innovation and Technology and the province of Salzburg under contract number 825345.

\section{References}

1. Bunt, B.: Obliquereflections: softwareart \& the 3d gamesengine. In: CyberGames 2006, Perth, Western Australia (2006)

2. Alexander, C., Ishikawa, S., Silverstein, M.: A Pattern Language: Towns, Buildings, Constructions (Center for Environmental Structure Series). University Press, Oxford (1977)

3. Gamma, E., Helm, R., et al.: Design Patterns: Elements of Reusable Object-Oriented Software. Pearson Education Corporate Sales Devision, Indianapolis (1995)

4. Obrist, M., Wurhofer, D., et al.: CUX patterns approach: Towards contextual user experience patterns. In: PATTERNS 2010, Lisbon, Portugal (2010)

5. Remo, C., http://www.gamasutra.com/view/feature/4325/past_and_ future_tension_the_.php

6. El-Nasr, M.: Projectingtension in virtualenvironmentsthroughlighting. In: CHI 2006, Montréal, Québec, Canada (2006)

7. Ravaja, N., Salminen, M., et al.: Emotional responsepatternsand sense ofpresenceduringvideogames: Potential criterion variables forgame design. In: NordiCHI 2004, Tampere, Finland (2004)

8. Ståhl, A., Höök, K., Sundström, P.: A Foundation for Emotional Expressivity. In: DUX 2005, San Francisco, USA (2005)

9. Von Ehrenfels, C.: Übergestaltqualitäten. Vierteljahrsschriftfürwissenschaftliche Philosophie. $\mathrm{n} / \mathrm{a}(1890)$

10. McCrea, C.: Horror Video Games: Essays on the Fusion of Fear and Play. McFarland \& Company, Inc., Publishers, North Carolina (2009)

11. Welton, J.: Eyewitness Art - Looking at Paintings. Dorling Kindersley, London (1994)

12. El-Nasr, M., Zupko, J., Miron, K.: Intelligent lightingfor a bettergamingexperience. In: CHI 2005, Portland, Oregon, USA (2005)

13. Marschall, S.: Farbeim Kino. SchürenVerlag GmbH, Marburg (2009)

14. Eisemann, E., Assarsson, U., et al.: Casting shadows in real time. In: SIGGRAPH ASIA 2009, Yokohama, Japan (2009)

15. Schaap, R., Bidarra, R.: Towards emotional characters in computergames. In: ICEC 2009, Taipei, Taiwan (2009)

16. Björk, S., Holopainen, J.: Patterns in Game Design. Charles River Media, Inc., Hingham (2005)

17. Power, M., Dalgleish, T.: Cognition and Emotion: From Order to Disorder. Psychology Press Ltd., Hove (1997)

18. El-Nasr, M., Yan, S.: Visual attention in 3d videogames. In: ACE 2006, San Antonio, Texas, USA (2006)

19. Jie, L., Clark, J.: Game Design Guided by Visual Attention. In: Ma, L., Rauterberg, M., Nakatsu, R. (eds.) ICEC 2007. LNCS, vol. 4740, pp. 345-355. Springer, Heidelberg (2007) 